\title{
Ultrasonic Absorption of Polycarbonate in Dioxane and in Chloroform
}

\author{
Shigeo Kato, Hiroyasu Nomura, and Yutaka Miyahara \\ Department of Chemical Engineering, Faculty of Engineering, \\ Nagoya University, Chikusa-ku, Nagoya 464, Japan.
}

(Received January 10, 1977)

\begin{abstract}
Measurements of ultrasonic absorption were carried out for polycarbonate in dioxane and in chloroform at frequencies from 1 to $130 \mathrm{MHz}$ over a temperature range of 10 to $55^{\circ} \mathrm{C}$. The molecular weight of the sample was about $4.0 \times 10^{4}$. In both systems double relaxation processes were observed. For the first relaxation process (at a lower frequency) the relaxation frequencies of polycarbonate in solutions do not differ very much from those of vinyl-type polymers in spite of structural differences in the backbone chains. The first relaxation process is attributed to the local segmental motions concerned with several monomer units or more. The second relaxation process (at a higher frequency) in solutions of polycarbonate and of vinyl-type polymers are strongly affected by solvents.

KEY WORDS Polymer Solution / Ultrasonic Absorption / Polycarbonate / Relaxation Time/ Segmental Motion /
\end{abstract}

In our previous papers, ${ }^{1-4}$ we reported the measurements of the ultrasonic absorptions of vinyl-type polymers such as polystyrene in various solvents in the frequency range from 1 to $100 \mathrm{MHz}$. Ultrasonic relaxation processes were observed in these polymer solutions. These relaxation processes were represented either with a single relaxation equation which has the relaxation frequency at several $\mathrm{MHz}$ or with the double relaxation equation which has an additional relaxation at a higher frequency. Many arguments have been presented as to whether the relaxation process of vinyl-type polymers in solutions are double or single in the frequency range from 1 to $100 \mathrm{MHz}$. However, it is agreed by all that there is the existence of a relaxation process for the lower frequency. From the experimental results we consider that the mechanism for this relaxation process is thermal and attributable to local segmental motions of polymers concerned with several units of monomers or more. If the relaxation process at the lower frequency is involved with only a few monomer units of a polymer chain, the relaxation behavior at several $\mathrm{MHz}$ could alter with a change in the skeleton of the polymers. In order to elucidate this relaxation mechanism and to obtain more information about the local segmental motions of polymers in solutions, studies of polymers having long monomer units and short side chains are necessary. Polycarbonate consists of the long units $\mathrm{C}-\phi-\mathrm{C}$ and of short side chains. In this paper, results of the measurements on the ultrasonic absorptions of polycarbonate in dioxane and in chloroform will be presented and the relaxation mechanisms discussed.

\section{EXPERIMENTAL AND SAMPLE}

The apparatus and the measuring procedures of ultrasonic absorption have been reported previously. ${ }^{5}$ The frequency range measured was from 1 to $130 \mathrm{MHz}$ and temperature range from 0 to $55^{\circ} \mathrm{C}$. The ultrasonic velocities were measured with the ultrasonic interferometer working at $4 \mathrm{MHz}$. The densities of solutions were measured with an Ostwald type pycnometer of $20 \mathrm{ml}$ in content. The commercial product of poly(2,2-bis ( $p$-hydroxyphenyl)propane carbonate) was used. The molecular weight of the 
sample was about $4.0 \times 10^{4}$. Dioxane was purified by the distillation after dehydration. The spectra grade reagent of chloroform was used without further purification.

Measurements were made at the following concentration; $1.68 \mathrm{~g} / 100 \mathrm{~m} l$ in dioxane and 4.03 $\mathrm{g} / 100 \mathrm{~m} l$ in chloroform.

\section{RESULTS}

\section{Solution in Dioxane}

Figure 1 shows the ultrasonic absorption coefficients of polycarbonate in solution at $25^{\circ} \mathrm{C}$ plotted as a function of frequency. As is apparent in Figure 1, the single relaxation equation fails to describe the relaxation behavior of polycarbonate in dioxane. This leads one to consider the following double relaxation equation

$$
\frac{\Delta \alpha}{f^{2} \cdot C}=\frac{A_{1} / C}{1+\left(f / f_{\mathrm{r} 1}\right)^{2}}+\frac{A_{2} / C}{1+\left(f / f_{\mathrm{r} 2}\right)^{2}}+B / C
$$

where $C, f_{\mathrm{r} 1}, f_{\mathrm{r} 2}$ and $\Delta \alpha / f^{2}$ are the concentration, relaxation frequencies and the difference

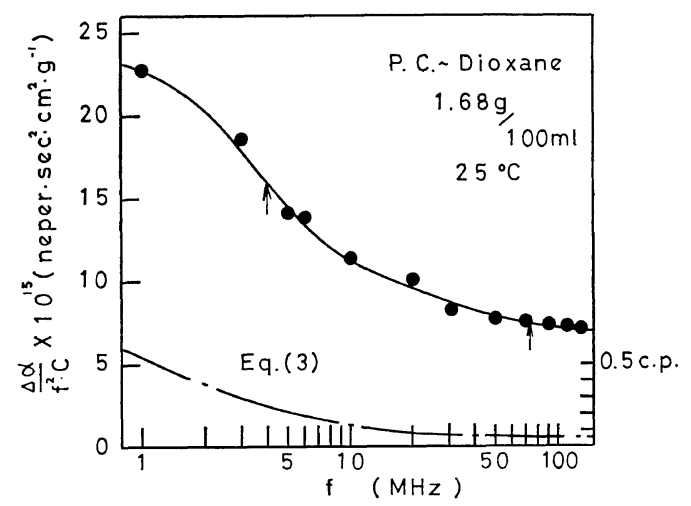

Figure 1. Ultrasonic absorption of polycarbonate in dioxane at $25^{\circ} \mathrm{C}$ : - double relaxation; -.., calculated curve from eq 3 .

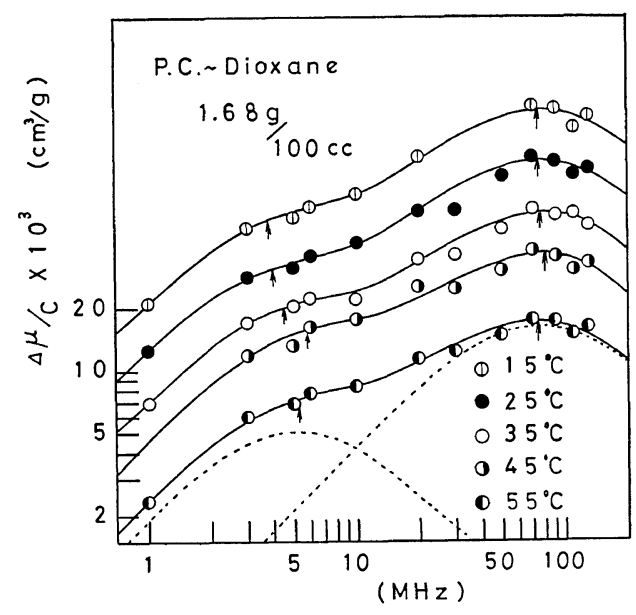

Figure 2. Excess absorption per wave length for polycarbonate in dioxane: (1) $15^{\circ} \mathrm{C} ; 0,25^{\circ} \mathrm{C} ; \bigcirc$, $35^{\circ} \mathrm{C} ; 15^{\circ} \mathrm{C} ; \bigcirc, 55^{\circ} \mathrm{C} ;-\ldots$, relaxation process.

in ultrasonic absorption coefficients $\Delta \alpha / f^{2}=$ $\alpha / f_{\text {solution }}^{2}-\alpha / f_{\text {solvent }}^{2}$, respectively. $A_{1}, A_{2}$, and $B$ are the relaxation parameters.

Figure 2 shows the relationship between absorption maxima per wave length and frequencies at various temperatures. In order to avoid the overlapping of the curves, the ordinate is shifted by equi-interval. The acoustic parameters describing the relaxation behavior of eq 1 are given in Table I.

\section{Solution in Chloroform}

Figure 3 shows the ultrasonic absorption coefficients of polycarbonate in chloroform plotted as a function of frequency. In this system the experimental values are also represented satisfactorily by the double relaxation equation. It should be emphasized that the ultrasonic absorption of pure solvent at $40^{\circ} \mathrm{C}$ is larger than that of the solution and the difference $\Delta \alpha / f^{2}$ is

Table I. Relaxation parameters for polycarbonate in dioxane

\begin{tabular}{cccccccc}
\hline$t,{ }^{\circ} \mathrm{C}$ & $\begin{array}{c}A_{1} / C \times 10^{15}, \\
\mathrm{neper} \cdot \mathrm{sec}^{2} \\
\mathrm{~cm}^{2} \cdot \mathrm{g}^{-1}\end{array}$ & $\begin{array}{c}A_{2} / C \times 10^{15} \\
\text { neper } \mathrm{cm}^{2} \cdot \mathrm{g}^{-1}\end{array}$ & $\begin{array}{c}B / C \times 10^{15} \\
\mathrm{ceper}^{15} \mathrm{sec}^{2}\end{array}$ & $\begin{array}{c}f_{\mathrm{r} 1}, \\
\mathrm{MHz}\end{array}$ & $\begin{array}{c}f_{\mathrm{r} 2}, \\
\mathrm{MHz}\end{array}$ & $\begin{array}{c}\mu_{1} / C \times 10^{3}, \\
\mathrm{~cm}^{3} \cdot \mathrm{g}^{-1}\end{array}$ & $\begin{array}{c}\mu_{2} / C \times 10^{3}, \\
\mathrm{~cm}^{3} \cdot \mathrm{g}^{-1}\end{array}$ \\
\hline 15 & 13.3 & 3.7 & 6.0 & 3.8 & 74 & 3.54 & 19.2 \\
25 & 14.3 & 3.7 & 5.5 & 4.0 & 74 & 3.94 & 18.8 \\
35 & 15.2 & 3.7 & 5.5 & 4.5 & 76 & 4.47 & 18.4 \\
45 & 16.6 & 3.7 & 5.9 & 5.7 & 80 & 5.96 & 18.7 \\
55 & 16.4 & 3.6 & 5.5 & 5.2 & 74 & 5.10 & 16.4 \\
\hline
\end{tabular}


Ultrasonic Absorption of Polycarbonate

Table II. Relaxation parameters for polycarbonate in chloroform

\begin{tabular}{rccccccc}
\hline$t,{ }^{\circ} \mathrm{C}$ & $\begin{array}{c}A_{1} / C \times 10^{15}, \\
\mathrm{neper} \cdot \mathrm{sec}^{2} \\
\mathrm{~cm}^{2} \cdot \mathrm{g}^{-1}\end{array}$ & $\begin{array}{c}A_{2} / C \times 10^{15}, \\
\mathrm{neper} \cdot \mathrm{sec}^{2} \\
\mathrm{~cm}^{2} \cdot \mathrm{g}^{-1}\end{array}$ & $\begin{array}{c}B / C \times 10^{15}, \\
\mathrm{neper} \cdot \mathrm{sec}^{2} \\
\mathrm{~cm}^{2} \cdot \mathrm{g}^{-1}\end{array}$ & $\begin{array}{c}f_{r 1}, \\
\mathrm{MHz}\end{array}$ & $\begin{array}{c}f_{r 2}, \\
\mathrm{MHz}\end{array}$ & $\begin{array}{c}\mu_{1} / C \times 10^{3}, \\
\mathrm{~cm}^{3} \cdot \mathrm{g}^{-1}\end{array}$ & $\begin{array}{c}\mu_{2} / C \times 10^{3}, \\
\mathrm{~cm}^{3} \cdot \mathrm{g}^{-1}\end{array}$ \\
\hline 0 & 18.6 & 11.4 & 62.6 & 6.0 & 84 & 5.97 & 51.2 \\
10 & 18.4 & 12.4 & 68.0 & 7.0 & 92 & 6.66 & 59.2 \\
20 & 18.1 & 10.9 & 72.7 & 7.0 & 94 & 6.35 & 51.4 \\
30 & 15.4 & 10.4 & 76.9 & 8.5 & 94 & 6.33 & 47.4 \\
40 & 15.9 & 11.9 & 77.9 & 9.0 & 100 & 6.66 & 55.5 \\
\hline
\end{tabular}

Table IlI. Activation energies for various systems

\begin{tabular}{|c|c|c|c|c|}
\hline Polymer & Solvent & $\underset{\mathrm{Kcal} \cdot \mathrm{mol}^{-1}}{\Delta H_{\mathrm{app}} 1,}$ & $\underset{\mathrm{Kcal} \cdot \mathrm{mol}^{-1}}{\Delta H_{\mathrm{app}}{ }^{2}}$ & Ref. \\
\hline P.V.Bu. & Toluene & $2.48 \pm 0.12$ & $0.70 \pm 0.01$ & (4) \\
\hline P.V.Pr. & Toluene & $1.30 \pm 0.16$ & $0.12 \pm 0.02$ & (4) \\
\hline P.V.P. & D.M.F. & $1.76 \pm 0.30$ & $0.53 \pm 0.28$ & $(15)$ \\
\hline P.C. & Dioxane & $1.85 \pm 0.33$ & $0.15 \pm 0.14$ & \multirow{2}{*}{ This work } \\
\hline P.C. & Chloroform & $1.70+0.17$ & $0.63 \pm 0.09$ & \\
\hline
\end{tabular}

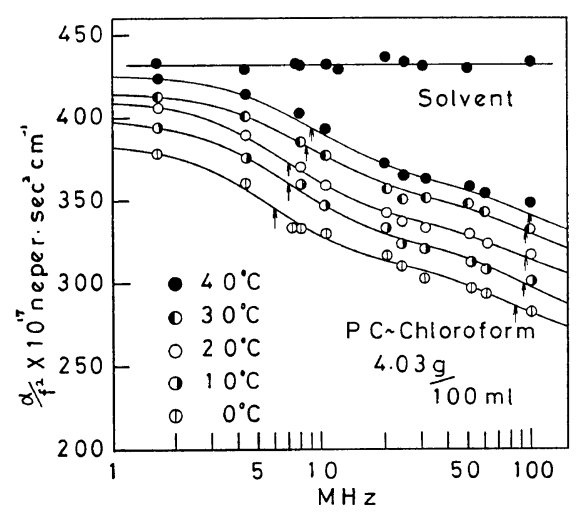

Figure 3. Frequency dependence of ultrasonic absorption of polycarbonate in chloroform: (1), $0^{\circ} \mathrm{C}$; (), $10^{\circ} \mathrm{C} ; \mathrm{O}, 20^{\circ} \mathrm{C} ; \mathrm{O}, 30^{\circ} \mathrm{C} ; \mathrm{O}, 40^{\circ} \mathrm{C}$.

negative. This tendency was observed at all temperatures and frequencies in these experiments. The acoustic parameters describing the relaxation behavior are given in Table II. In this system the analysis of the relaxation behavior was done by the following equation.

$$
\frac{\alpha}{f^{2} \cdot C}=\frac{A_{1} / C}{1+\left(f / f_{\mathrm{r} 1}\right)^{2}}+\frac{A_{2} / C}{1+\left(f / f_{\mathrm{r} 2}\right)^{2}}+B / C
$$

Figure 4 shows the relationship between relaxation frequencies and reciprocal temperature. The activation energies estimated from Figure 4

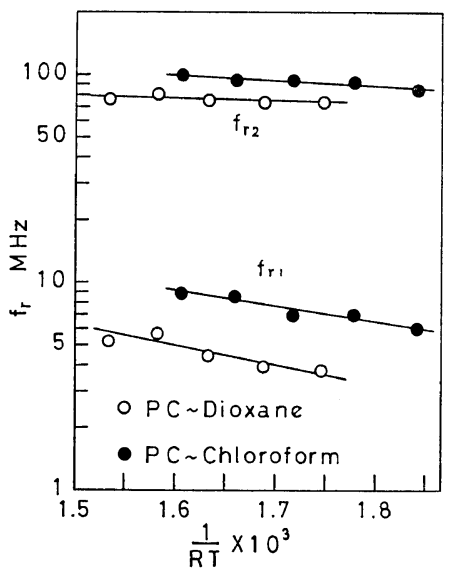

Figure 4. Relationship between relaxation frequencies and reciprocal temperature: O, P.C.dioxane; O, P.C.-chloroform.

are given in Table III.

\section{DISCUSSION}

Contribution of Shear Viscosity to the Ultasonic Absorption

As is well known, the absorption of longitudinal waves in liquid is generally expressed as follows;

$$
\frac{\alpha}{f^{2}}=\frac{2 \pi^{2}}{\rho u^{3}}\left\{\eta_{\mathrm{v}}{ }^{\prime}(\omega, T)+\frac{4}{3} \eta_{\mathrm{s}}^{\prime}(\omega, T)\right\}
$$


where $\eta_{\mathrm{v}}{ }^{\prime}$ is the dynamic volume viscosity and $\eta_{\mathrm{s}}{ }^{\prime}$ is the dynamic shear viscosity. $\rho$ and $u$ are density and sound velocity, respectively.

Assuming that the energy dissipation of polymer solutions is due only to the friction of beads against solvent, Okano ${ }^{6}$ derived the relation $\eta_{\nabla}{ }^{\prime}=(2 / 3) \eta_{\mathrm{s}}{ }^{\prime}$ for the volume viscosity of polymer solutions. With this relation, and the relaxation times ${ }^{7}$ of dynamic shear modulus obtained from Zimm's theory, it is possible to calculate the absorption of acoustic longitudinal waves in polymer solutions from eq 3 . The result of the calculation is shown in Figure 1. Figure 1 shows that friction makes only slight contributions to the ultrasonic absorption in this range. Gotlib, et al., ${ }^{8}$ have recently explained the ultrasonic absorption in polymer solutions using a friction model in which the local viscosity is taken into account. Their model can success fully foretell the relaxation frequency but it explains only a small portion of the relaxation strength. According to Ferry, et al. ${ }^{9}$ the dynamic shear viscosity of polymer solutions is larger than that of pure solvent at the limit of high frequency. On the other hand a very different behavior is observed in the relaxation process of longitudinal acoustic waves. Namely, in such systems as polycarbonatechloroform and poly(methyl methacrylate)-benzene, ${ }^{10}$ differences $\Delta \alpha / f^{2}$ are negative.

As pointed out by Ohsawa ${ }^{11}$ and Ono, ${ }^{12}$ the facts mentioned above suggest that greater part of the ultrasonic absorption in the present frequency range should be attributed to something other than shear viscosity only.

\section{Relaxation Mechanisms of Polycarbonate in Solution}

As mentioned above, two relaxation processes are observed in each of the two solutions of polycarbonate. The first relaxation process exists in the frequency range from several to $10 \mathrm{MHz}$ and the second, from 70 to $100 \mathrm{MHz}$.

First Relaxation Process. Several models have been proposed to explain the mechanism of the first relaxation process for the vinyl-type polymers in solutions. ${ }^{12-14}$ We believe that this relaxation process is a thermal one which is attributable to the local motion of polymer chains concerned with several units of monomers.

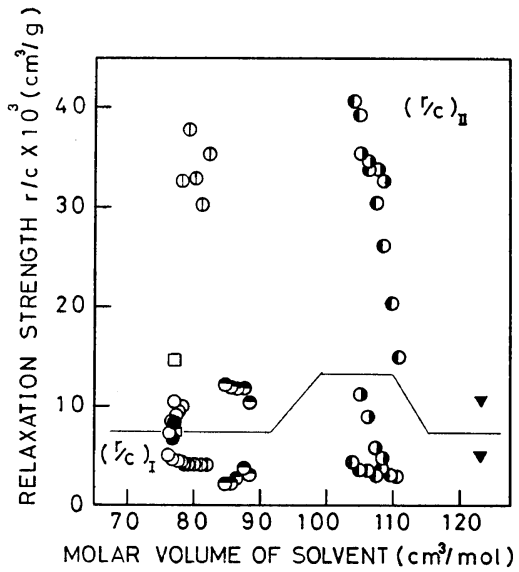

Figure 5. Relationship between relaxation strengths and molar volumes of solvents: $\Theta$, P.C.-dioxane; (1) P.C.-chloroform; O, P.V.Bu.-toluene; ${ }^{4}$, P.V.Pr.-toluene; ${ }^{4}$ O, P.V.P.-D.M.F.; ${ }^{15}$ O, P.S.D.M.F.;22 $\square$, P.S.-D.M.F.;21 $\nabla$, P.S. -xylene. ${ }^{12}$

Ono, et al., ${ }^{12}$ have proposed a similar relaxation mechanism, assuming the volume relaxation predominantly. According to them, the volume relaxation was ascribed to the exchange of solvent molecules and a positive correlation was found between the relaxation strength and the molar volume of solvent.

Figure 5 shows the relationship between the relaxation strength and the molar volume of solvent. As is seen in Figure 5, the relaxation strengths of the first relaxation process are nearly constant and independent of the molar volume of solvent. Therefore the relaxation process of polycarbonate in solutions can not be explained by the "solvation" model.

Thoughs much experimentations are still necessary for further clarification of the relaxation mechanism of polycarbonate in solutions, we feel, at present, that the relaxation mechanism is as follows; Polycarbonate is a semi-flexible polymer. However it has many free rotational parts because the long units, $\mathrm{C}-\phi-\mathrm{C}$, exist in its backbone chain and the steric hinderance between the side groupes is small. Therefore, a considerable degree in freedom of rotation is expected in its chain. In comparison with vinyltype polymers, the motion involving only one or two monomer units of polycarbonate is attended with much difficulty; that is, rotational 
motion is almost impossible in such units. However the aggregate consisting of several or more monomer units of polycarbonate is assumed to have some facility in rotation.

These considerations explain well the following features observed in this experiment.

1) For polycarbonate in solutions the first relaxation frequencies do not differ very much from those of vinyl-type polymers in solutions.

2) Activation energies do not differ very much from those of vinyl-type polymers in solutions (Table III).

3) The relaxation strengths of the first relaxation are independent of the molar volume of solvent. Their values are nearly the same (Figure 5).

Second Relaxation Process. As to the second relaxation process of the polymer solutions, there is the need to discuss its mechanism. At this stage, we point out only the following facts for the second relaxation process.

1) As the side chains of polycarbonate are small, the second relaxation process of polycarbonate in solutions may be attributed to the local segmental motion concerned with the smaller parts of the main chain. In this point Fünfshilling's model ${ }^{14}$ offers helpfull suggestions.

2) As is seen in Figure 5 the relaxation strengths of the second relaxation process depend strongly on the solvent for the polycarbonate as well as do also the vinyl-type polymer in solution. Therefore the mechanisms bringing about solvent effects should be considered.

In chloroform and $N, N$-dimethylformamide, the apparent activation energies of the second relaxation process are larger than those in other systems (Table 3). Poly(vinyl pyrrolidone) shows the double relaxation process in $N, N$-dimethylformamide $^{15}$ while it shows the single relaxation process in water. ${ }^{16}$ In various solvents polystyrene also shows similar behavior as does also poly(vinyl pyrrolidone). The above can be explained by considering the strong solvent effects on the second relaxation process.

\section{Solvent Solute Interaction of Polycarbonate in Chloroform}

In polycarbonate chloroform system the ultrasonic absorption coefficients are lower than those of the solvent at any frequency in this work.
Such behavior is unusual in polymer solutions. Another example is the system of poly(methyl methacrylate)-benzene reported by Tondre, et al. ${ }^{10}$ Some reports ${ }^{17,18}$ show the existence of the strong solvent solute interaction in chloroform. The negative $\Delta \alpha / f^{2}$ may also be attributed to the solvent solute interactions. Though no other detailed information has been given on the origin of the negative $\Delta \alpha / f^{2}$, we propose the following very crude model, assuming that the differences of $\alpha / f^{2}$ between solvent and solution are entirely attributable to the shift of the relaxation frequency to a higher frequency. The shift of the relaxation frequency of chloroform is estimated as follows;

The relaxation strength of vibrational relaxation $r$ is given by the following equation

$$
r=\frac{(\gamma-1) \Delta C_{\mathrm{vib}}}{\left(C_{\mathrm{p}}-\Delta C_{\mathrm{vib}}\right)}
$$

where $C_{\mathrm{p}}$ and $\gamma$ are the specific heat at constant pressure and the ratio of specific heats, respectively. $\Delta C_{\mathrm{vib}}$ is the contribution of the molecular vibrations to the static specific heat. In a dilute solution, the vibrational heat capacity of solution has the same value as that of the solvent. Therefore the relaxation strengths are nearly equal in both systems. Assuming that the relaxation behavior of chloroform is the single relaxation in $\mathrm{GHz}$ region, we obtain

$$
\frac{A}{A^{\prime}} \doteqdot \frac{f_{\mathrm{r}}^{\prime}}{f_{\mathrm{r}}}
$$

where $A, A^{\prime}, f_{\mathrm{r}}$ and $f_{\mathrm{r}}{ }^{\prime}$ are relaxation parameters and relaxation frequencies of solvent and solution, respectively. This method predicts well the relaxation frequencies of binary mixtures of Kneser liquids. ${ }^{20}$ For example, in $\mathrm{CS}_{2}-\mathrm{CCl}_{4}$ mixtures, we obtain $f_{\mathrm{r}}{ }^{\prime} \doteqdot 116 \mathrm{MHz}\left(\mathrm{CS}_{2} 99 \%\right)$ and $248 \mathrm{MHz}\left(\mathrm{CS}_{2} 95 \%\right)$, while the observed values are $110 \mathrm{MHz}$ and $210 \mathrm{MHz}$, respectively. By putting $A^{\prime}$ equal to $B$ of the polycarbonatechloroform system, $f_{\mathrm{r}}^{\prime}$ is estimated from eq 5 at $20^{\circ} \mathrm{C}$,

$$
f_{\mathrm{r}}{ }^{\prime} \doteqdot 1.36 \times 10^{9} \mathrm{~Hz}
$$

where the literature values for $A$ and $f_{\mathrm{r}}^{19}$ are used. Further discussion about solvent solute interactions will be made at a later date, following further experimentation. 


\section{CONCLUSION}

1) Measurements of ultrasonic absorption were carried out for polycarbonate in dioxane and in chloroform over a frequency range from 1 to $130 \mathrm{MHz}$. In both systems, double relaxation processes were observed.

2) For the first relaxation process (at a lower frequency), the relaxation frequencies of polycarbonate in solution do not differ very much from those of vinyl-type polymers in spite of the structural differences in the backbone chains. The first relaxation process is attributed to the local segmental motions concerned with several monomer units or more.

3) The second relaxation process (at the higher frequency side) in solutions of vinyl-type polymers and of polycarbonate is strongly affected by solvents.

\section{REFERENCES}

1. H. Nomura, S. Kato, and Y. Miyahara, Nippon Kagaku Zasshi, 91, 837 (1970).

2. H. Nomura, S. Kato, and Y. Miyahara, $J$. Material Soc. Jpn., 20, 669 (1971).

3. H. Nomura, S. Kato, and Y. Miyahara, Nippon Kagaku Kaishi, 1973, 1554.

4. H. Nomura, S. Kato, and Y. Miyahara, ibid., 1973, 2398.

5. H. Nomura and Y. Miyahara, Nippon Kagaku Zasshi, 88, 502 (1967).

6. K. Okano, Rept. Prog. Polym. Phys. Jpn., 5, 67 (1962).
7. G. Harrison, J. Lamb, and A. J. Matheson, J. Phys. Chem., 68, 1972 (1964).

8. Y. Y. Gotlib and A. A. Darinskii, Soviet. Phys. Acoust., 19, 430 (1974).

9. D. J. Massa, J. L. Schrag, and J. D. Ferry, Macromolcules, 4, 210 (1971).

10. C. Tondre and R. Cerf, J. Chim. Phys., 65, 1105 (1968).

11. T. Ohsawa and Y. Wada, Polym. J., 1, 465 (1970).

12. K. Ono, H. Shintani, O. Yano, and Y. Wada, ibid., 5, 164 (1973).

13, H. Hässler and H. J. Bauer, Kolloid-Z. $Z$. Polym., 230, 194 (1969).

14. O. Fünfshilling, P. Lemaréchal, and R. Cerf, Chem. Phys. Lett., 365 (1971).

15. S. Kato, I. Uehara, H. Kondo, H. Nomura, and Y. Miyahara, Nippon Kagaku Kaishi, 1975, 1651.

16. S. Kato, H. Kondo, I. Fujio, H. Nomura, and Y. Miyahara, ibid., 1974, 1981.

17. S. Teramachi, A. Takahashi and I. Kagawa, Nippon Kogyo Kagaku Zasshi, 69, 685 (1966).

18. L. W. Reeves and W. G. Schneider, Can. J. Chem., 35, 251 (1957).

19. L. A. Davidovichi, A. A. Ivanov, S. Makhkamov, L. Pulatova, P. K. Khabibullaev., M. G. Khaliulin, and Sh. Sharipov, Soviet. Phys. Acoust., 19, 18 (1973).

20. J. L. Hunter, D. Dossa, J. Haus, and D. Sette, J.C.P., 60, 4605 (1974).

21. W. Ludlow, E. Wyn-Jones, and J. Rassing, Chem. Phys. Lett., 13, 477 (1972).

22. P. Lemaréchal, ibid., 16, 495 (1972). 